\title{
Fluctuations of the Unruh Temperature f
}

\author{
JEAN-GUY DEMERS \\ Center for Theoretical Physics, \\ Laboratory for Nuclear Science \\ and Department of Physics, \\ Massachusetts Institute of Technology \\ Cambridge, Massachusetts 02139 U.S.A. \\ email: jgdemers@mitlns.mit.edu
}

\begin{abstract}
Using the influence functional formalism, the problem of an accelerating detector in the presence of a scalar field in its ground state is considered in Minkowski space. As is known since the work of Unruh, to a quantum mechanical detector following a definite, classical acceleration, the field appears to be thermally excited. We relax the requirement of perfect classicality for the trajectory and substitute it with one of derived classicality through the criteria of decoherence. The ensuing fluctuations in temperature are then related with the time and the amplitude of excitation in the detector's internal degree of freedom.
\end{abstract}

MIT-CTP 2342

August 1994

${ }^{*}$ This work is supported in part by funds provided by the U. S. Department of Energy (D.O.E.) under cooperative agreement \#DE-FC02-94ER40818. 
The influence functional (IF) formalism was developed 30 years ago by Feynman and Vernon[1] to study the properties of quantum open systems. It has since been usefully applied to a number of physical problems (including masers, polaron, transport, localization) and was also shown to be closely related with the close-time-path formalism[2]. More recently, it was used by quantum cosmologists to study the conditions under which a quantum mechanical system decoheres and can be said to behave classically owing to its interaction with the unobserved environment[3]. The use of the IF in that context can be seen in the evolution of the reduced density matrix.

Suppose the system and the environment have each a single degree of freedom, that they are described by the actions $S_{s y s}[x]$ and $S_{\text {env }}[y]$ respectively and that their interaction is described by the action $S_{\text {int }}[x, y]$. Suppose also that at $t=0$, the system and the environment are not correlated so that the total density matrix is separable:

$$
\rho\left(x_{1}, y_{1}, x_{2}, y_{2} ; t=0\right)=\rho_{\text {sys }}\left(x_{1}, x_{2} ; t=0\right) \times \rho_{\text {env }}\left(y_{1}, y_{2} ; t=0\right) \text {. }
$$

Then at time $t$ later, the reduced density matrix (where the environment is integrated out) will be given by [4, 0$]$ :

$$
\rho_{\text {red }}\left(x_{1}^{f}, x_{2}^{f} ; t\right)=\int d x_{1}^{i} \int d x_{2}^{i} P\left(x_{1}^{f}, x_{2}^{f}, t \mid x_{1}^{i}, x_{2}^{i}, 0\right) \rho_{\text {sys }}\left(x_{1}^{i}, x_{2}^{i} ; 0\right),
$$

with the kernel

$$
P\left(x_{1}^{f}, x_{2}^{f}, t \mid x_{1}^{i}, x_{2}^{i}, 0\right)=\int_{x_{1}^{i}}^{x_{1}^{f}} \mathcal{D} x_{1} \int_{x_{2}^{i}}^{x_{2}^{f}} \mathcal{D} x_{2} e^{i\left(S_{s y s}\left[x_{1}\right]-S_{s y s}\left[x_{2}\right]\right)} e^{i \Gamma\left[x_{1}, x_{2}\right]},
$$

where the boundary conditions on the functional integrals are given for times 0 and $t$ (our units are such that $\hbar=c=1$ ). The IF is the last term in (3). It is a property of the environment and the the way it is coupled to the system and its formal expression is

$$
\begin{gathered}
e^{i \Gamma\left[x_{1}, x_{2}\right]}=\int d y^{f} d y_{1}^{i} d y_{2}^{i} \int_{y_{1}^{i}}^{y^{f}} \mathcal{D} y_{1} \int_{y_{2}^{i}}^{y^{f}} \mathcal{D} y_{2} e^{i\left(S_{\text {env }}\left[y_{1}\right]+S_{\text {int }}\left[x_{1}, y_{1}\right]\right)} \\
\times e^{-i\left(S_{\text {env }}\left[y_{2}\right]+S_{\text {int }}\left[x_{2}, y_{2}\right]\right)} \rho_{\text {env }}\left(y_{1}^{i}, y_{2}^{i} ; 0\right)
\end{gathered}
$$




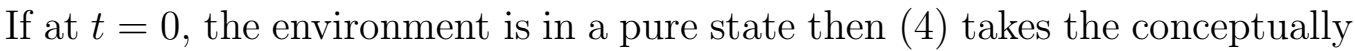
simple form

$$
e^{i \Gamma\left[x_{1}, x_{2}\right]}=\left\langle\psi_{2}(t) \mid \psi_{1}(t)\right\rangle .
$$

Here, $\left|\psi_{1}(t)\right\rangle$ is the state that evolved from the initial state at $t=0$ under the dynamics dictated by $S_{\text {env }}\left[y_{1}\right]+S_{i n t}\left[x_{1}, y_{1}\right]$ in which $x_{1}$ acts as a c-number, time dependant source; likewise $\left|\psi_{2}(t)\right\rangle$ is governed by $S_{\text {env }}\left[y_{2}\right]+S_{\text {int }}\left[x_{2}, y_{2}\right]$. For an environment made of many non-interacting and initially uncorrelated particles, it is easy to see that the total $\Gamma$ is simply the sum of the contributions from each particle. Unless the paths $\left[x_{1}(t)\right]$ and $\left[x_{2}(t)\right]$ are such that the states in (5) are adiabaticlly disturbed, $\Gamma$ will have not only a real part $\Gamma_{R}$ but also a (positive) imaginary part $\Gamma_{I}$.

In the next section, we review how the IF predicts the heating of the scalar field forming the environment of a detector on a uniformly accelerating course [6, 7]. This is achieved by assuming that while the detector's internal degree of freedom is quantum mechanical, its position degree of freedom remains strictly classical. In Section 3 , we consider a different limit. The spacetime trajectory is taken to be a decohered spacetime path, with a spread around the uniformly accelerating trajectory. Focusing on this position degree of freedom and taking now the detector's excitation to be fixed, we obtain a relation for the spread in acceleration involved in constructing the approximately classical path.

As usual, environment-induced decoherence is indicated by the diagonalization of the reduced density matrix. Thus, in the position basis, decoherence at a time $\mathrm{t}$ is a measure of how $\rho_{\text {red }}\left(x_{1}^{f}, x_{2}^{f} ; t\right)$ has become diagonal. It is clear that if $\Gamma_{I}$ is large for pair of paths $\left(x_{1}(t), x_{2}(t)\right)$ that are far apart from one another in spacetime, then the contribution of these pairs will be suppressed in (3). As a result, $\rho_{\text {red }}\left(x_{1}^{f}, x_{2}^{f} ; t\right)$ will be more diagonal. Our study of decoherence will thus be through $\Gamma_{I}$. Note that a decoherence functional is sometimes defined to include coarse graining of the system paths in addition to the tracing out of the environment [ [ [ []. But in that case, diagonalization of the IF defined here is still the mechanism to achieve decoherence. In the last section, we remark on our result and comment on their relevance in the context of black holes. 
Consider an environment made of a massless scalar field in two spacetime dimensions [5, 7]. In a finite box of spatial dimension $L$, the mode expansion reads

$$
\phi(x, t)=\sqrt{\frac{2}{L}} \sum_{k}\left[y_{k}^{+} \cos k x+y_{k}^{-} \sin k x\right],
$$

with $k=2 \pi n / L$ with $n=1,2 \ldots$. The kinetic term is

$$
\begin{aligned}
L_{e n v} & =\int d x \frac{1}{2} \partial_{\mu} \phi \partial^{\mu} \phi \\
& =\frac{1}{2} \sum_{\sigma=+,-} \sum_{k}\left[\left(\dot{y}_{k}^{\sigma}\right)^{2}-k^{2}\left(y_{k}^{\sigma}\right)^{2}\right],
\end{aligned}
$$

where dots denote time derivative. The system is formed by a detector that is linearly and locally sensitive to the matter field with an interacting Lagrangian density

$$
\mathcal{L}_{\text {int }}(x)=-\varepsilon Q \phi(x, t) \delta(x-X(t))
$$

where $\varepsilon$ is a coupling constant while $Q(t)$ is the DeWitt monopole moment 9] and plays the role of $x(t)$ in Section 11. $X(t)$ is the position of the detector which is assumed here to follow a classical, definite trajectory in time. Performing the trivial spatial integration gives

$$
\begin{aligned}
L_{i n t} & =-\varepsilon Q \phi(X(t), t), \\
& =-\varepsilon Q \sqrt{\frac{2}{L}} \sum_{k}\left[y_{k}^{+} \cos k X(t)+y_{k}^{-} \sin k X(t)\right], \\
& \equiv-\sum_{k \sigma} Q c_{k}^{\sigma}(t) y_{k}^{\sigma},
\end{aligned}
$$

where

$$
\begin{aligned}
c_{k}^{+}(t) & =\varepsilon \sqrt{\frac{2}{L}} \cos k X(t), \\
c_{k}^{-}(t) & =\varepsilon \sqrt{\frac{2}{L}} \sin k X(t) .
\end{aligned}
$$


In this way, the IF for the observed dipole degree of freedom $Q$ is computed from a set of forced, non-relativistic harmonic oscillators with a time dependent coupling constant whose evolution depends on the trajectory of the detector. But as it is well known, the Feynman propagator for that case may be computed exactly [10]. As a result, the IF (5) can also be obtained and one gets

$$
\begin{aligned}
\Gamma_{R}\left[Q_{1}, Q_{2}\right] & =-\int_{0}^{t} d s_{1} \int_{0}^{s_{1}} d s_{2}\left[Q_{1}\left(s_{1}\right)-Q_{2}\left(s_{1}\right)\right] \mu\left(s_{1}, s_{2}\right)\left[Q_{1}\left(s_{2}\right)+Q_{2}\left(s_{2}\right)\right], \\
\Gamma_{I}\left[Q_{1}, Q_{2}\right] & =\int_{0}^{t} d s_{1} \int_{0}^{s_{1}} d s_{2}\left[Q_{1}\left(s_{1}\right)-Q_{2}\left(s_{1}\right)\right] \nu\left(s_{1}, s_{2}\right)\left[Q_{1}\left(s_{2}\right)-Q_{2}\left(s_{2}\right)\right],
\end{aligned}
$$

where $\mu\left(s_{1}, s_{2}\right)$ and $\nu\left(s_{1}, s_{2}\right)$ are respectively the noise and dissipation kernels and depend on $c_{k}^{\sigma}(t)$. If the detector is following a trajectory with uniform acceleration $a$ :

$$
X(\tau)=\frac{1}{a} \cosh (a \tau), \quad t(\tau)=\frac{1}{a} \sinh (a \tau),
$$

where $\tau$ is the proper time, then in that case one can show that 5

$$
\begin{aligned}
& \mu\left(\tau_{1}, \tau_{2}\right)=-\int_{0}^{\infty} d \omega I(\omega) \sin \omega\left(\tau_{1}-\tau_{2}\right), \\
& \nu\left(\tau_{1}, \tau_{2}\right)=\int_{0}^{\infty} d \omega I(\omega) \operatorname{coth} \frac{\pi \omega}{a} \cos \omega\left(\tau_{1}-\tau_{2}\right)
\end{aligned}
$$

where $I(\omega)=\frac{\varepsilon^{2}}{2 \pi \omega}$ is the spectral density. But except for being written in proper time, the kernels (13) are just those obtained when the environment is taken to be a bath of harmonic oscillator at temperature

$$
k_{B} T=a / 2 \pi
$$

linearly coupled via an interaction term of the form $L_{i n t}=-\varepsilon \sum_{k \sigma} Q y_{k}^{\sigma}[1$, , 11]. In this way, the heating of the vacuum as sensed by an accelerating detector is apparent in the IF formalism 1 .

\footnotetext{
${ }^{1}$ One can also define the interacting Lagrangian to incorporate an integral over proper time and a delta function over inertial time (see Ref. [7] ). In that setup, the time integrals in (11) will come out in proper time. In view of next section where the proper time will not be unique, we remain here in inertial time.
} 


\section{3}

In displaying the Unruh effect in the last section, it was assumed that the position degree of freedom of the detector was not quantum mechanical, as the detector followed a well defined, classical trajectory. Although this was sufficient to extract the basic physics of the heating of the vacuum, we know that this classicality is an approximation. From a path integral point of view, this treatment would require that the uniformly accelerating trajectory sufficiently decohere from other neighboring paths to form a consistent history 2 . But of course the decoherence is always finite at a given time. We now consider the uncertainty associated with the fluctuations in the detector's paths around the uniformly accelerating trajectory.

For simplicity, we will take the limit where the value of the monopole degree of freedom $Q$ is given by its quantum mechanical average. In view of the approximately stationary nature of the acceleration, this average will also be assumed to be constant in time. As stated in Section 1, our criteria for decoherence will be $\Gamma_{I}$. This quantity involves the specification of two paths. To study the extent to which the acceleration is well defined, we take two uniformly accelerating trajectories with slightly different accelerations:

$$
x_{i}=\sqrt{a_{i}^{-2}+t^{2}}
$$

for $i=1,2$. After defining,

$$
\begin{aligned}
& g_{1 k}^{+} \equiv \varepsilon Q \sqrt{\frac{2}{L}} \cos k \sqrt{a_{1}^{-2}+t^{2}} \\
& g_{1 k}^{-} \equiv \varepsilon Q \sqrt{\frac{2}{L}} \sin k \sqrt{a_{1}^{-2}+t^{2}} \\
& g_{2 k}^{+} \equiv \varepsilon Q \sqrt{\frac{2}{L}} \cos k \sqrt{a_{2}^{-2}+t^{2}} \\
& g_{2 k}^{-} \equiv \varepsilon Q \sqrt{\frac{2}{L}} \sin k \sqrt{a_{2}^{-2}+t^{2}}
\end{aligned}
$$

\footnotetext{
${ }^{2}$ Although sufficient deoherence is usually not the only criteria for classicality, it is nevertheless essential, and will be our focus here (see for example Refs. [12, 15]).
} 
we have for the Lagrangians (77) and (9) the result [5]:

$$
\begin{aligned}
\Gamma_{I}\left(a_{1}, a_{2}, t\right)= & \frac{1}{2} \sum_{\sigma=+,-} \sum_{k} \int_{0}^{t} d s_{1} \int_{0}^{s_{1}} \frac{d s_{2}}{k}\left[g_{1 k}^{\sigma}\left(s_{1}\right)-g_{2 k}^{\sigma}\left(s_{1}\right)\right] \\
& \cos \left(s_{1}-s_{2}\right)\left[g_{1 k}^{\sigma}\left(s_{2}\right)-g_{2 k}^{\sigma}\left(s_{2}\right)\right], \\
= & \frac{\varepsilon^{2} Q^{2}}{2 \pi} \int_{0}^{t} d s_{1} \int_{0}^{s_{1}} d s_{2} \int_{0}^{\infty} \frac{d k}{k}[\cos k A+\cos k B \\
= & \frac{\varepsilon^{2} Q^{2}}{8 \pi} \int_{0}^{t} d s_{1} \int_{0}^{s_{1}} d s_{2} P\left(s_{1}, s_{2}, a_{1}, a_{2}\right) .
\end{aligned}
$$

In the second equality of (17), we have done the sum over $\sigma$, defined the quantities

$$
\begin{aligned}
A & \equiv \sqrt{a_{1}^{-2}+s_{1}^{2}}-\sqrt{a_{1}^{-2}+s_{2}^{2}}, \\
B & \equiv \sqrt{a_{2}^{-2}+s_{1}^{2}}-\sqrt{a_{2}^{-2}+s_{2}^{2}}, \\
C & \equiv \sqrt{a_{1}^{-2}+s_{1}^{2}}-\sqrt{a_{2}^{-2}+s_{2}^{2}}, \\
D & \equiv \sqrt{a_{2}^{-2}+s_{1}^{2}}-\sqrt{a_{1}^{-2}+s_{2}^{2}}, \\
F & \equiv s_{1}-s_{2},
\end{aligned}
$$

and took the continuum limit for $k$ by substituting $\sum_{k} \rightarrow \frac{L}{2 \pi} \int_{0}^{\infty} d k$. In the last equality of (17), the $k$ integration was performed by making use of 13

$$
\int_{0}^{\infty} d x \frac{\sin a x \sin b x}{x}=\frac{1}{2} \ln \left|\frac{a+b}{a-b}\right|
$$

and we also defined

$$
P\left(s_{1}, s_{2}, a_{1}, a_{2}\right) \equiv \ln \left(\frac{C^{2}-F^{2}}{A^{2}-F^{2}} \frac{D^{2}-F^{2}}{B^{2}-F^{2}}\right)^{2} .
$$

Note that in (20), as $s_{2} \rightarrow s_{1}, A^{2}-F^{2}$ and $B^{2}-F^{2}$ vanish and $P$ is divergent on that "line". Depending on the values of $s_{1}, a_{1}, a_{2}$, there will generally also be values of $s_{2}$ for which $C^{2}-F^{2}$ or $D^{2}-F^{2}$ are vanishing. But since those divergencies are logarithmic, the decoherence remains nevertheless finite, even 
without imposition of a ultra-violet cut-off. This is in contrast with what is usually done in the Caldeira-Legget model [11] and in the minisuperspace approximation of quantum cosmology (see for example [15]).

We now wish to extract the features of $\Gamma_{I}$ for paths lasting a time large compared with the inverse accelerations $\left(t a_{1} \gg 1, t a_{2} \gg 1\right)$. Consider the rate of increase of $\Gamma_{I}$ with time. From (17),

$$
\dot{\Gamma}_{I}\left(t, a_{1}, a_{2}\right)=\frac{\varepsilon^{2} Q^{2}}{8 \pi} t R\left(t, a_{m}, a_{d}\right),
$$

with:

$$
\begin{aligned}
R\left(t, a_{m}, a_{d}\right) & \equiv \int_{0}^{1} d y P\left(s_{1}=t, s_{2}=y t, a_{1}, a_{2}\right) \\
& =R\left(\tilde{t}, \tilde{a}_{d}\right) .
\end{aligned}
$$

In (21), we made the change of variable $s_{2}=y t$ ( $y$ is unitless), and worked with the average $a_{m} \equiv \frac{1}{2}\left(a_{1}^{-1}+a_{2}^{-1}\right)$ and difference $a_{d} \equiv\left(a_{1}^{-1}-a_{2}^{-1}\right)$ in inverse accelerations. In the last line of (22), $a_{m}$ was used for the scale factor in $P$, namely: $\tilde{t} \equiv t / a_{m}$ and $\tilde{a_{d}} \equiv a_{d} / a_{m}$. Now except for a transient time $\tilde{t} \lesssim 1$, we have

$$
R\left(\tilde{t}, \tilde{a}_{d}\right) \rightarrow f\left(\tilde{a}_{d}\right)
$$

that is, $R$ is time independent to a good approximation. (Obviously, $f\left(\tilde{a}_{d}\right)$ is an even function.) This can be seen in Fig.1, where numerical evaluations of $R$ are given as a function of $\tilde{t}$ for a few representative values of $\tilde{a}_{d}$. Note that for $\tilde{t}<1, R$ takes large values, and in fact diverge as $\tilde{t} \rightarrow 0$, since in (20), $A^{2}-F^{2}$ and $B^{2}-F^{2}$ vanish in that limit. But when multiplied by $t$ in (21), $\dot{\Gamma}_{I}$ remains obviously finite. For example, at $\tilde{a}_{d}=0.1$, the peak in $t R$ occurs for $\tilde{t} \approx \tilde{a}_{d}$ and has height 0.7 . As $\tilde{t}$ grows beyond unity, the time development of $\dot{\Gamma}_{I}$ is clearly linear to a very good approximation. In the computation of $\dot{\Gamma}_{I}$, the importance of the initial peak becomes negligible in the limit of small $\tilde{a}_{d}$ and large $\tilde{t}$. In that regime, (21) integrates to

$$
\Gamma_{I}\left(t, a_{1}, a_{2}\right) \approx \frac{\varepsilon^{2} Q^{2}}{16 \pi} t^{2} f\left(\tilde{a}_{d}\right) .
$$

Using (22), (with (20)), a simple and rough estimate can be obtained for $f\left(\tilde{a}_{d}\right)$ by the substitutions

$$
\sqrt{\left(1 \pm \frac{1}{2} \tilde{a}_{d}\right)^{2}+\tilde{t}^{2}} \rightarrow \tilde{t}
$$




$$
\sqrt{\left(1 \pm \frac{1}{2} \tilde{a}_{d}\right)^{2}+\tilde{t}^{2} y^{2}} \rightarrow \tilde{t} y
$$

after multiplying out the terms in (20) (e.g. $C^{2}-F^{2} \rightarrow 2 a_{m}^{2}+\frac{1}{2} a_{d}^{2}$ ). Eq. (25) is clearly a poor substitution around $y=0$ and also fails to reproduce any of the divergencies present in $P$ mentioned above. To leading order in $\tilde{a}_{d}$, it leads to $f\left(\tilde{a}_{d}\right)=2 \tilde{a}_{d}^{2}$. The actual decoherence is in fact larger than what this predicts. But as $\tilde{a}_{d}$ gets smaller, it is in fact quite good, up to a multiplicative constant $N$. The Fig.2 presents the exact, numerically computed result for $f\left(\tilde{a}_{d}\right)$ in the range $10^{-4}<\tilde{a}_{d}<10^{-1}$ (obtained with $\tilde{t}=10$ ). It also includes the curve $N \tilde{a}_{d}^{2}$ for $N=10$ and $N=110$. It is seen that $f\left(\tilde{a}_{d}\right)$ approaches a quadratic behavior as $\tilde{a}_{d}$ decreases. With (24) we thus have for $\left|\tilde{a}_{d}\right| \ll 1$ and $\tilde{t} \gg 1$ the approximation

$$
\Gamma_{I}\left(t, a_{1}, a_{2}\right) \approx \frac{N}{16 \pi} \varepsilon^{2}\langle Q\rangle^{2} t^{2}\left(\frac{a_{1}-a_{2}}{\left(a_{1}+a_{2}\right) / 2}\right)^{2},
$$

where $Q$ was replaced by its quantum mechanical average (quantum fluctuations of $Q$ are neglected).

We now take the condition $\Gamma_{I} \approx 1$ as separating the decohering and nondecohering accelerations. Using (26) and (14), we conclude that a detector on a decohered accelerating trajectory for a time $t$ will be subject to thermal fluctuations of the order:

$$
\left|\frac{\Delta T}{T}\right| \approx \frac{1}{|\varepsilon|\langle Q\rangle t}
$$

where we dropped a constant of order one. Eq. (27) can also be understood as an uncertainty relation between the 'time of flight' of the detector and the spread in temperature, with the uncertainty given by $\frac{1}{|\varepsilon|\langle Q\rangle}$ and valid for $t / T>1$.

\section{4}

In Eq. (26), the quadratic dependance of the decoherence on the coupling to the bath was already apparent from (16). It is general for a system coupled linearly to the bath (by construction, the amplitude of the detector's excitations act to modify the effective coupling). It is interesting to see that for the class of paths considered here, the dependance in time is also quadratic. 
This shows that the time is also making the coupling constant stronger or inversely, that a larger coupling constant is identical to a longer time evolution. Qualitatively, this is in line with the result of Ref. [14]. As the coupling to the bath gets stronger and that one waits longer, clearly more particles are created and decoherence is increased. This can also be seen through (5). When particles are copiously produced, a slight difference in the two paths will easily make the two states orthogonal.

Issues of decoherence similar to the ones considered here could well prove to be relevant in the context of black holes. As it is well known, one of the distinguishing features of black holes is their ability to Hawking radiate, in close connection with the uniformly accelerating detector. The environment given by these radiated particles could then serve in the study of the validity of the semi-classical approximation 15, 16.

\section{ACKNOWLEDGMENTS}

I am grateful to Samir Mathur for a number of discussions in relation with this work. 


\section{References}

[1] R. Feynman and F. L. Vernon, Ann. Phys. 24 (1963) 118.

[2] Z. Su, L.-Y. Chen, X. Yu and K. Chou, Phys. Rev. B37 (1988) 9810, and references therein; E. Calzetta and B.L. Hu, Phys. Rev. D35 495 (1987). On the Close-Time-Path formalism, see the reviews: N. P. Landsman and Ch. van Weert, Phys. Rep. 145 (1987) 141 ; K. Chou, Z. Su, B. Hao and L. Yu, Phys. Rep. 118 (1985) 1.

[3] W. H. Zurek, Phys. Rev. D24 1516 (1981); Phys. Rev. D26 186 (1982); Phys. Today 44 (1991) 36; M. Gell-Mann and J. B. Hartle, in Complexity, Entropy and the Physics of Information, edited by W. H. Zurek, Santa Fe Institute Studies in the Sciences of Complexity Vol. VIII (Addison-Wesley, Reading, MA, 1990).

[4] H. F. Dowker and J. J. Halliwell, Phys. Rev. D46 (1992) 1580.

[5] B. L. Hu and A. Matacz, Maryland U. preprint UMDPP-93-210.

[6] W. G. Unruh, Phys. Rev. D14 (1976) 870.

[7] J. R. Anglin, Phys. Rev. D47 (1993) 4525.

[8] J. B. Hartle, in Quantum Cosmology and Baby Universes, Proceedings of the 7th Jerusalem Winter School, edited by S. Coleman, J. Hartle, T. Piran and S. Weinberg, Jerusalem, Israel, 1990, (World Scientific, Singapore, 1991).

[9] B. S. DeWitt, in General Relativity: An Einstein Centennial Survey, edited by S. W. Hawking and W. Israel (Cambridge University Press, Cambridge, England, 1979), p. 680.

[10] R. Feynman and C. R. Hibbs, Quantum Mechanics and Path Integrals, (McGraw-Hill, New-York, 1965)

[11] A. O. Caldeira and A. J. Legget, Physica A121 (1983) 587.

[12] J. J. Halliwell, Phys. Rev. D48 (1993) 4785. 
[13] I. S. Gradshteyn and I. M. Ryzhik, Tables of Integrals, Series, and Products, 4th ed. (Academic, San Diego, CA, 1980), p. 414, Eq. 3-741(1).

[14] E. Calzetta and D. Mazzitelli, Phys. Rev. D42 (1990) 4066.

[15] J.P. Paz and S. Sinha, Phys. Rev. D44 (1991) 1038; Phys. Rev. D45 (1992) 2823

[16] See for example J. B. Hartle, in Gravitation in Astrophysics (Cargese 1986), Proceedings of the Summer Institute, Cargese, France, 1986, edited by B. Carter and J. Hartle, NATO ASI Series B: Physics, Vol. 156 (Plenum, New York, 1987); T. Padmanabhan, Class. Quantum Grav. 6 (1989) 533; T. Padmanabhan and T.P. Singh, Class. Quantum Grav. 7 (1990) 411; S. D. Mathur, MIT-CTP-2304 (hep/th 9404135); E. KeskiVakkuri, G. Lifschytz, S. D. Mathur and M. Ortiz, MIT-CTP-2341. 


\section{Figure Captions}

Fig.1 : Plot of $R\left(\tilde{t}, \tilde{a}_{d}\right)$ as a function of the reduced time $\tilde{t}$, for $\tilde{a}_{d}=0.05$ (curve A), $\tilde{a}_{d}=0.075$ (curve $\mathbf{B}$ ) and $\tilde{a}_{d}=0.1$ (curve $\mathbf{C}$ ). It is apparent that (for a given $\tilde{a}_{d}$ ) as $\tilde{t} \gtrsim 1, R\left(\tilde{t}, \tilde{a}_{d}\right)$ is well approximated by a constant.

Fig.2 : Logarithmic plot of $f\left(\tilde{a}_{d}\right)$ (curve $\mathbf{A}$ ) for a range of $\tilde{a}_{d}$. The function $N \tilde{a}_{d}^{2}$ is also presented for $N=110$ (curve B) and $N=10$ (curve C). As $\tilde{a}_{d}$ decreases, the behavior of $f\left(\tilde{a}_{d}\right)$ becomes nearly quadratic. 
This figure "fig1-1.png" is available in "png" format from: http://arxiv.org/ps/gr-qc/9408016v1 
This figure "fig1-2.png" is available in "png" format from: http://arxiv.org/ps/gr-qc/9408016v1 